\begin{tabular}{|l|c|c|c|c|}
\hline Cuadernos I. Geográfica & 24 & pp. 39-57 & Logroño & 1998 \\
\hline
\end{tabular}

\title{
LA GESTIÓN DE LOS USOS DEL SUELO COMO ESTRATEGIA PARA MEJORAR LA PRODUCCIÓN Y LA CALIDAD DEL AGUA. RESULTADOS EXPERIMENTALES EN EL PIRINEO CENTRAL ESPAÑOL
}

\author{
T. LASANTA MARTINEZ ${ }^{1}$ \\ J. M. GARCIA RUIZ
}

\begin{abstract}
RESUMEN: Los diferentes usos del suelo y cubiertas vegetales proporcionan una amplia gama de valores de escorrentía superficial y de concentración de sedimentos. En la Estación Experimenal "Valle de Aísa", localizada en el Pirineo Central español, se ba obtenido información sobre el funcionamiento bidromorfológico de los usos del suelo tradicionales y actuales, confirmando que el abandono de tierras de cultivo en laderas pendientes y la expansión de los prados y del matorral representan un descenso en la producción de sedimento y en la disponibilidad de agua a escala de cuenca. Con esta perspectiva se discuten posibles cambios de uso del suelo que contribuyan a mejorar la calidad de los recursos bídricos.

ABSTRACT: Different land-uses and plant covers yield a wide range of overland flow and sediment concentration. In the "Aísa Valley" Experimental Station, located in the Central Spanish Pyrenees, information has been obtained on the bydromorpbological functioning of both actual and traditional land-uses, confirming that farmland abandonment in steep slopes and the expansion of meadows and shrub cover represent a decrease in both
\end{abstract}

1 Instituto Pirenaico de Ecología, CSIC, Campus de Aula Dei, Apartado 202, 50.080 Zaragoza. e-mail: fm@ipe.csic.es

2 Instituto Pirenaico de Ecología, CSIC, Campus de Aula Dei, Apartado 202, 50.080 Zaragcra. e-mail: humberto@ipe.csic.es 
sediment yield and water availability at a basin scale. With this in mind the authors discuss possible land-use changes that contribute to improve the quality of water resources.

Palabras clave: erosión, usos del suelo, calidad del agua, montaña, Pirineos Key words: erosion, land uses, water resources quality, mountain areas, Pyrenees

\section{Introducción}

La calidad y disponibilidad de los recursos hídricos constituye el problema ambiental más importante en muchos países del mundo. El elevado contenido de material en suspensión en el agua reduce espectacularmente la vida activa de los embalses, tanto para regadío como para la producción de energía eléctrica, causando igualmente problemas en las llanuras aluviales. A la vez, la escasez de recursos hídricos (a veces sólo estacional) está en el origen de problemas sociales y económicos que demandan grandes inversiones y son algunas de las razones de la baja calidad de vida de millones de personas.

Los recursos hídricos en las regiones mediterráneas dependen principalmente de las áreas de montaña, que actúan como "islas de humedad" en medio de zonas más secas. Por ello, los cambios que afectan a la cubierta vegetal y a los usos del suelo en las montañas mediterráneas tienen consecuencias muy importantes en extensas áreas fuera de las montañas. Esta dependencia respecto de los recursos hídricos de las montañas es una cuestión muy importante, ya que las montañas mediterráneas han cambiado intensamente sus usos del suelo en las últimas décadas, con fuertes impactos sobre la dinámica fluvial (Martínez Castroviejo et al., 1991) y sobre la localización de las fuentes de sedimentos.

Es bien conocido el hecho de que la cantidad y calidad del agua disponible en una cuenca depende en parte de la cubierta vegetal y de los usos del suelo. Dunne \& Leopold (1978) ponen varios ejemplos sobre las posibilidades de incrementar la cantidad de agua y reducir la carga de sedimentos con una adecuada gestión de tierras. Sobre el papel hidrológico del bosque existen numerosos trabajos, que han sido sintetizados por Bosch \& Hewlett (1982). Sin embargo, es menos conocido el papel que cumplen las laderas cultivadas y de campos abandonados, así como los terrenos recolonizados por el matorral, a pesar de su amplia representación en montañas submediterráneas. En España destacan las aportaciones de Llorens (1993), Llorens et al. (1992 y 1997) sobre bancales abandonados, y las de Ruiz Flaño (1993) y García Ruiz et al. (1995) en laderas de campos abandonados en pendiente. El objetivo del presente trabajo 
es, en primer lugar, discutir, a partir de la información experimental disponible, el efecto de los usos del suelo sobre la calidad y cantidad de los recursos hídricos. En segundo lugar, se trata de definir las consecuencias hidromorfológicas de cambios de uso del suelo que pudieran proponerse para mejorar la gestión agroganadera.

\section{El área de estudio}

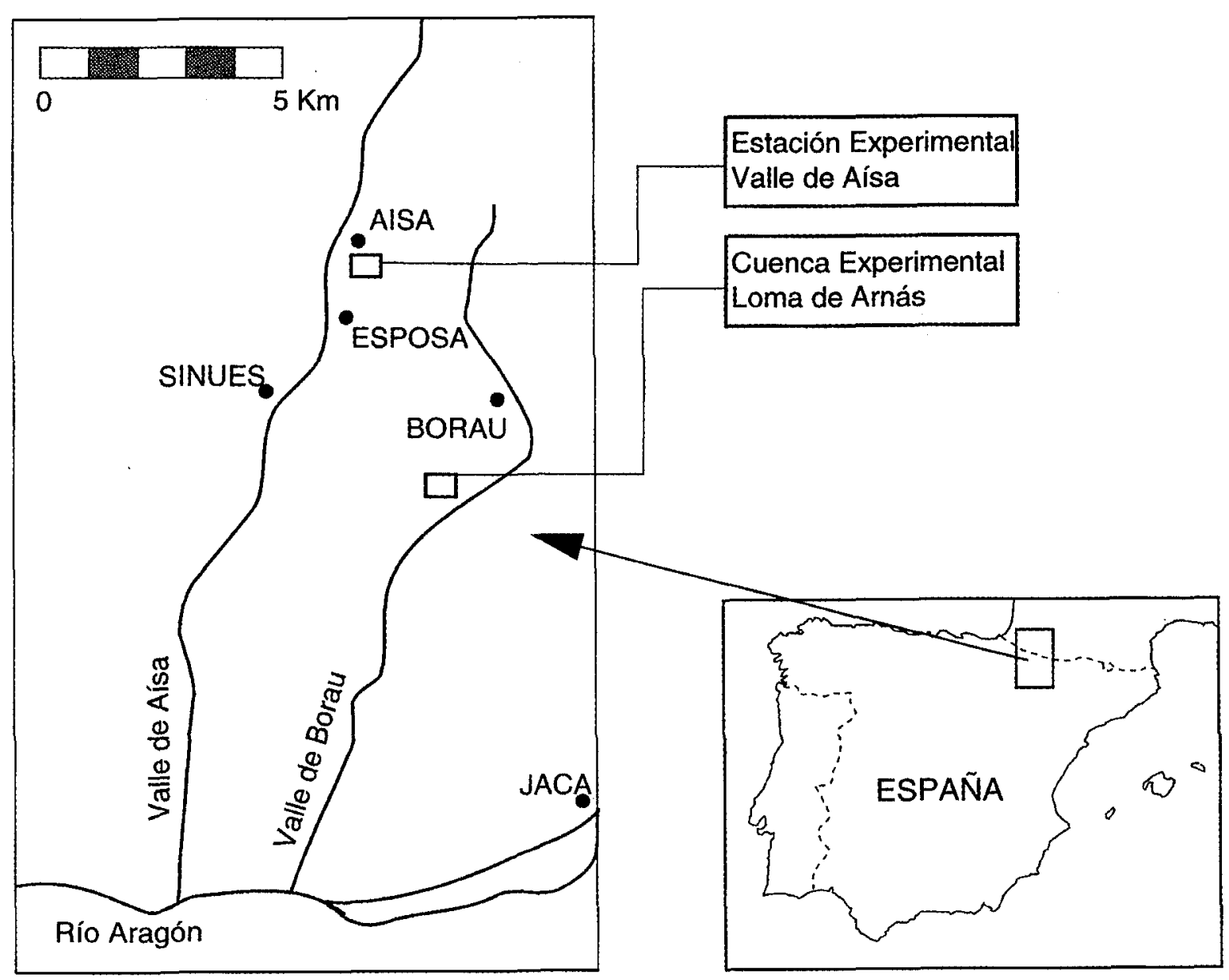

Fig. 1. Localización del área de estudio.

La parte experimental del estudio se ha llevado a cabo en el valle de Aísa, Pirineo Central español (Fig.1). Al norte predominan las calizas y areniscas de las Sierras Interiores, con grandes escarpes e importante impronta del glaciarismo pleistoceno. El resto del valle está ocupado por el flysch eoceno, con vertientes regularizadas y divisorias suaves. En la localidad de Aísa, a $1100 \mathrm{~m}$ s.n.m. la precipitación media anual es de $1100 \mathrm{~mm}$ y la temperatura media anual es de $10^{\circ} \mathrm{C}$. Por debajo de $1500 \mathrm{~m}$ los bosques de Pinus sylvestris domi- 
nan en las laderas umbrías, mientras en las solanas coexisten pequeños rodales de Quercus gr. faginea con diversos matorrales submediterráneos (especialmente Genista scorpius, Buxus sempervirens y Rosa gr. canina) y con repoblaciones de Pinus nigra. Por encima de $1500 \mathrm{~m}$ predominan los bosques densos de Pinus sylvestris. El piso subalpino (sobre 1700-1800 m) fue completamente deforestado desde la Edad Media para ampliar la superficie de pastos de verano. Los suelos son pardos, muy pedregosos y de escasa potencia. Son pobres en nutrientes y en materia orgánica (1.5\%) y ricos en carbonato. Su pH oscila entre 8 y 8.5 (Ruiz Flaño, 1993).

El valle de Aísa ocupa una superficie total de 8540 ha, de las que 1749 (el $23.8 \%$ de total) corresponden a la extensión del espacio agrícola en el momento de su máxima expansión (probablemente a mediados del siglo XIX). El cultivo principal era el cereal, en campos pendientes y exposición solana, a veces con barbechos muy largos, sobre todo en el caso de los sistemas agrícolas itinerantes (articas). Desde 1930 se han abandonado $1456 \mathrm{Ha}$. En la actualidad sólo se cultiva el fondo del valle, donde los cereales han sido sustituidos por cultivos forrajeros.

\section{Métodos}

La Estación Experimental "Valle de Aísa" se localiza cerca del pueblo de Aísa, a 1140 m s.n.m., en un campo abandonado hace 35 años, cubierto por matorral denso de Genista scorpius y Rosa gr. canina como especies más representativas. En 1991 se instalaron seis parcelas cerradas de $10 \times 3 \mathrm{~m}$, con un canal Gerlach en la parte baja de cada parcela conectado a un sistema de balancines y a data loggers para registrar los eventos de escorrentía de forma continua. Se instaló también un pluviógrafo conectado a uno de los data loggers para conocer el volumen e intensidad de la lluvia. Parte de la escorrentía de cada parcela es desviada desde los balancines a depósitos de entre 130 y 2201 de capacidad. Después de cada evento lluvioso se recoge una muestra de agua para conocer la exportación de sedimento en suspensión y materiales disueltos.

Cada una de las parcelas reproduce un uso del suelo: cereal de artigueo o sistema de agricultura nómada (cebada fertilizada con cenizas), barbecho, cereal (cebada fertilizada con abono químico), matorral (vegetación natural inalterada), prado y parcela de matorral incendiado.

En 1993 la parcela de barbecho pasó a cereal, mientras que la de cereal se dejó en rastrojo, iniciando un proceso de abandono. En el mismo año se instalaron dos nuevas parcelas, una dedicada a barbecho (que desde entonces alterna en uso con la de cereal) y una segunda de matorral incendiado. En 1996 se instaló otra parcela de artica, mientras que la inicial quedó abandonada. En la actualidad, hay pues, nueve parcelas (Fig. 2) que simulan los princi- 


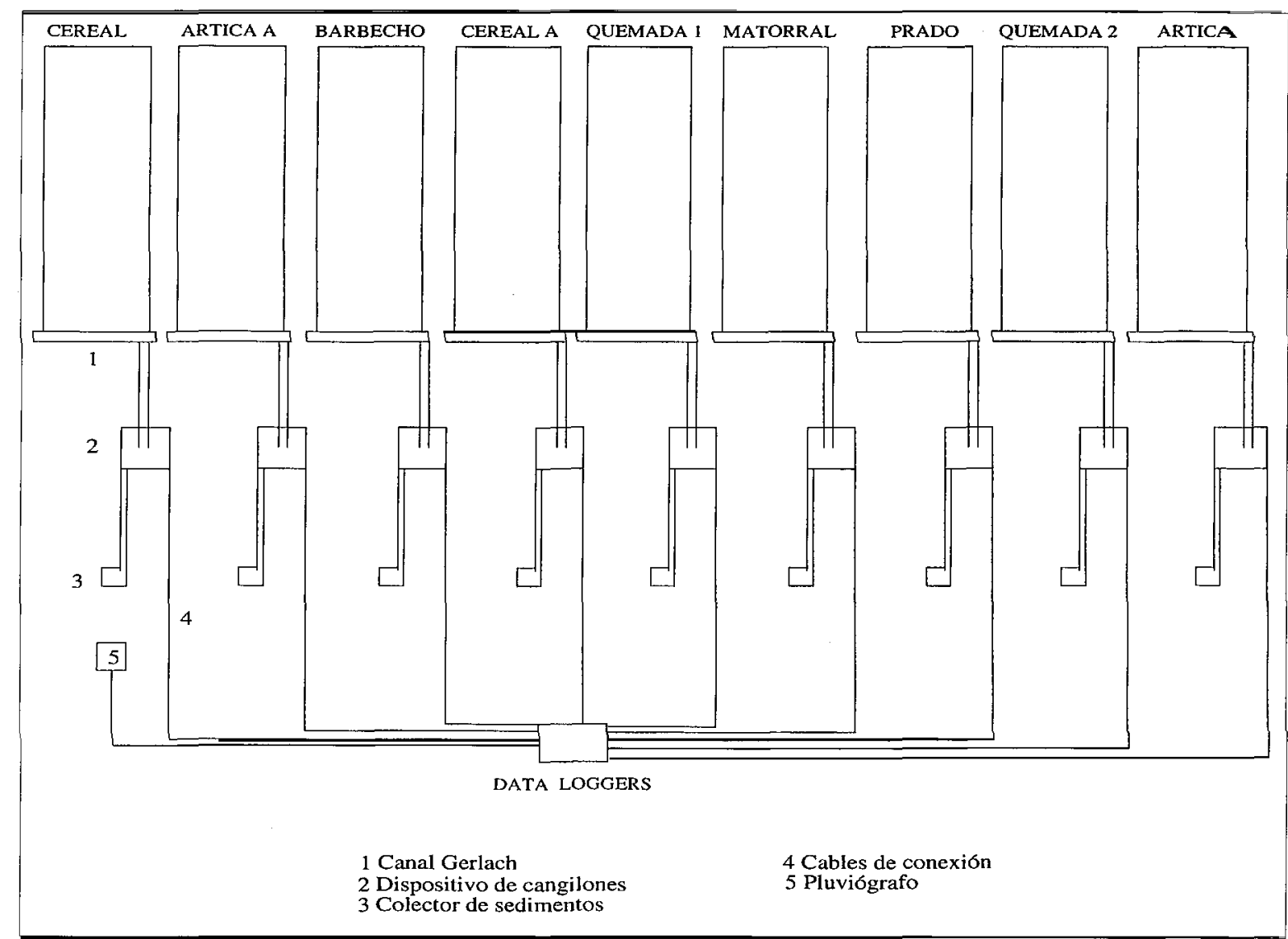

Fig. 2. La Estación Experimental "Valle de Aísa".

pales usos agroganaderos en los llamados sistema tradicional y actual de gestión del territorio en el Pirineo y, en general, en la mayoría de las montañas submediterráneas.

En 1996 se equipó la cuenca de Arnás (valle de Borau, inmediatamente al este del valle de Aísa) con el fin de obtener información sobre el caudal y el transporte de sedimento en un área muy afectada por la deforestación y el cultivo en laderas pendientes. Ocupa una superficie de 350 ha, la mayor parte de campos ya abandonados.

Finalmente, se realizó la cartografía de usos del suelo en el valle de Aísa, poniendo especial interés en la delimitación de los campos abandonados. Mediante el Sistema de Información Geográfica Arc/Info V7.04 se conoce su distribución en relación con diferentes factores topográficos.

\section{Resultados}

Diversos estudios han demostrado que durante el llamado sistema tradicional de uso del suelo la producción de sedimento fue mayor que en la actuali- 
dad (García Ruiz et al., 1995 y 1996; García Ruiz \& Valero Garcés, 1998). A principios del siglo XX muchos ríos mostraban un modelo trenzado inestable, con la llanura aluvial agradada por la acumulación de materiales heterométricos (Martínez Castroviejo et al., 1991). Se ha demostrado igualmente que el desarrollo de muchos conos de deyección está claramente relacionado con las actividades humanas (Gómez Villar, 1996). García Ruiz et al. (1996) sugieren que el cultivo de cereales en laderas pendientes fue responsable de la intensa erosión que ha afectado a muchas laderas solanas del Pirineo. Los mismos autores confirman que el abandono de tierras de cultivo y la reforestación explican la estabilización reciente de las estructuras sedimentarias fluviales (ver también Rubio \& Hernández, 1990), la contracción de las áreas más activas de los conos de deyección y el descenso en la producción de sedimento desde las laderas. Los usos del suelo aparecen, pues, en el centro de los grandes cambios geomorfológicos e hidrológicos ocurridos en el último siglo.

\subsection{La evolución de los usos del suelo}

La evolución de los usos agropecuarios del suelo en el valle de Aísa es similar a la registrada por los restantes valles del Pirineo Central (Lasanta, 1989). Durante siglos la actividad agrícola estuvo destinada a la producción de alimento para la población humana. El 37\% del espacio cultivado correspondía a sistemas nómadas (articas), sin técnicas de conservación del suelo. Los prados, localizados en conos de deyección, apenas tenían importancia (13\% de la superficie cultivada).

El ganado disponía de abundantes recursos forrajeros durante el verano, por encima del límite superior del bosque. Sin embargo, los recursos de invierno eran muy limitados (matorrales submediterráneos y rastrojos). Este desequilibrio entre invierno y verano es la base de la trashumancia pirenaica.

Desde 1950 se ha producido una rápida despoblación, que ha dado lugar a un progresivo abandono de la agricultura. Las laderas solanas, sobre suelos pobres, han sido completamente abandonadas, y la superficie cultivada actual se limita al fondo del valle, conos de deyección y rellanos colgados sobre depósitos glaciolacustres, todos ellos con pendientes suaves y suelos profundos, cerca de los pueblos y accesibles para la maquinaria (Lasanta, 1988). Además, los campos de cereal han sido sustituídos por prados, después de que la desaparición de la trashumancia obliga a incrementar la producción forrajera, limitando así la dependencia del exterior. En 1996 la cabaña ganadera está compuesta por 1821 ovejas, 144 cabras y 181 vacas. En 1942, cuando la trashumancia estaba vigente, se contabilizaron 6100 ovejas.

La mayor parte de los campos abandonados se han colonizado por matorrales. Sólo los campos que han continuado pastándose con cierta intensidad se 
mantienen como prados naturales o de diente (Molinillo et al., 1997). García Ruiz \& Lasanta (1993) calcularon que los campos abandonados contribuyen en un 5\% a la alimentación del ganado, frente al $66 \%$ de los campos de cultivo (prados) y el $27 \%$ de los pastos supraforestales. Estas cifras confirman la gran importancia que tiene el espacio cultivado actual para la supervivencia del sistema ganadero.

\subsection{Escorrentía y erosión en diferentes usos del suelo}

La Estación Experimental "Valle de Aísa" funciona desde 1991, si bien en este trabajo sólo se utiliza la información de los años 1996 y 1997 en los que no existe ningún vacío de datos. El año 1996 puede considerarse húmedo, con una precipitación total de $1,389 \mathrm{~mm}$, mientras el año 1997 estuvo cerca de la media, con 1,140 mm. La Fig. 3 muestra la distribución de la precipitación, destacando el periodo húmedo de finales de 1996: entre noviembre y diciembre se registraron $521 \mathrm{~mm}$. En 1997 noviembre fue también bastante húmedo (266 $\mathrm{mm}$ ), pero diciembre fue muy seco. Los valores de precipitación de primavera fueron bastante normales, si bien junio de 1996 fue muy seco y mayo de 1997 fue más húmedo que la media.

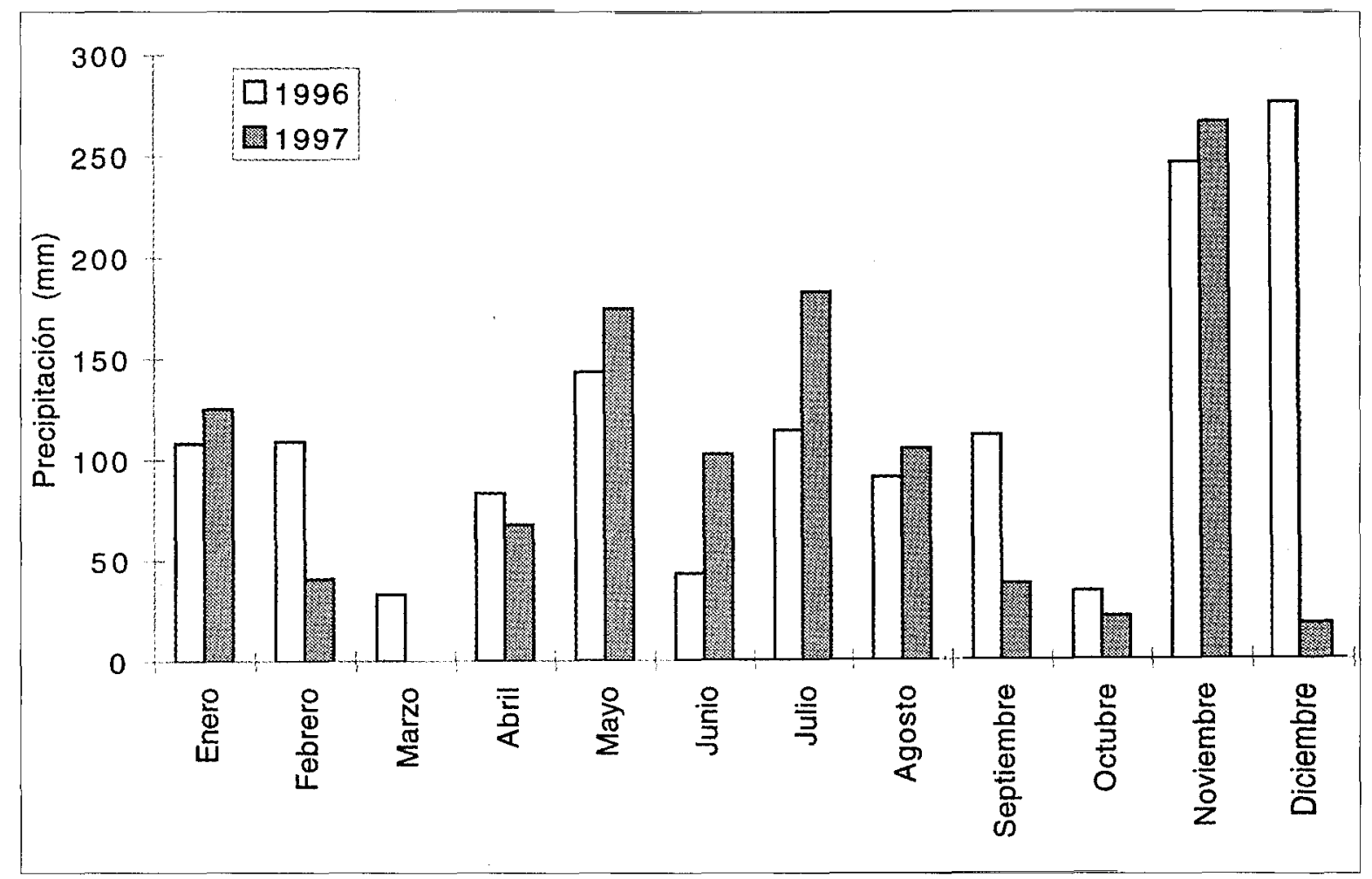

Fig. 3. Distribución mensual de la precipitación en 1996 y 1997. 
Los resultados obtenidos de las parcelas experimentales proporcionan información sobre las salidas de agua y las pérdidas de suelo (sedimento en suspensión y solutos. La Fig. 4 muestra los coeficientes de escorrentía, la concentración de sedimento y la pérdida de suelo en 1996, y la Fig. 5 incluye la misma información para 1997.

La primera impresión es que, aunque en general hay un buen ajuste entre los dos años, pueden observarse algunas diferencias. Estas últimas pueden deberse a dos razones. En primer lugar, el volumen total de precipitación y su distribución mensual son algo diferentes, lo que puede dar lugar a que los valores de exportación de agua y sedimento desde cada parcela experimenten algunas variaciones en función de la densidad de la cubierta vegetal en el momento de las lluvias más intensas. En segundo lugar, no debe olvidarse que el uso de parcelas experimentales para estimar la escorrentía y la pérdida de suelo está sujeto a muchas limitaciones. Las medidas en parcelas experimentales sólo son aceptables con fines comparativos, es decir, para disponer de ordenes de magnitud de la escorrentía y la erosión en diferentes ambientes y usos del suelo. En ningún caso pueden aceptarse como coeficientes absolutos o tasas. Es evidente que las características de las parcelas, el previsible agotamiento del sedimento, las modificaciones causadas en el suelo por su instalación y la interrupción artificial de la escorrentía reducen la validez de los datos, aunque los resultados son buenos indicadores de las diferencias entre los ambientes estudiados.

Las Figuras 4 y 5 muestran la existencia de varios grupos de parcelas:

i) El primer grupo incluye a la parcela quemada en 1991 y la parcela con matorral denso. Generan bajas cantidades de escorrentía superficial (coeficientes entre el 4 y el 6\%) y registran bajas concentraciones de sedimentos. En términos de erosión del suelo los valores obtenidos para los dos años son muy bajos (entre 9 y $12 \mathrm{~g} \cdot \mathrm{m}^{-2}$ ). En los dos casos la densidad de la cubierta vegetal fue muy alta tanto en el estrato arbustivo (Genista scorpius y Rosa gr. canina) como en el herbáceo (Carex flacca, Bracbipodium pinnatum y Bromus erectus como especies más representativas). La parcela quemada en 1991 recuperó pronto su cubierta vegetal y en 1996 presentaba ya el mismo aspecto que la parcela de matorral denso (no incendiada).

ii) La parcela de prado y la parcela abandonada en 1991 (previamente cultivada como cereal) tienen coeficientes de escorrentía más elevados que las parcelas anteriores especialmente la parcela abandonada. En ambas la concentración de sedimento es moderada (entre 157 y $258 \mathrm{mg}^{\mathrm{l}^{-1}}$ ), por lo que las pérdidas de suelo se incluyen también dentro de valores relativamente bajos (entre 12.4 y $28.3 \mathrm{~g}^{-2} \mathrm{~m}^{-2}$. Deben tenerse en cuenta, no obstante, dos consideraciones. En primer lugar, ambas parcelas se hallan cubiertas completamente por vegetación herbácea. Y en segundo lugar, la parcela abandonada ha soportado una 


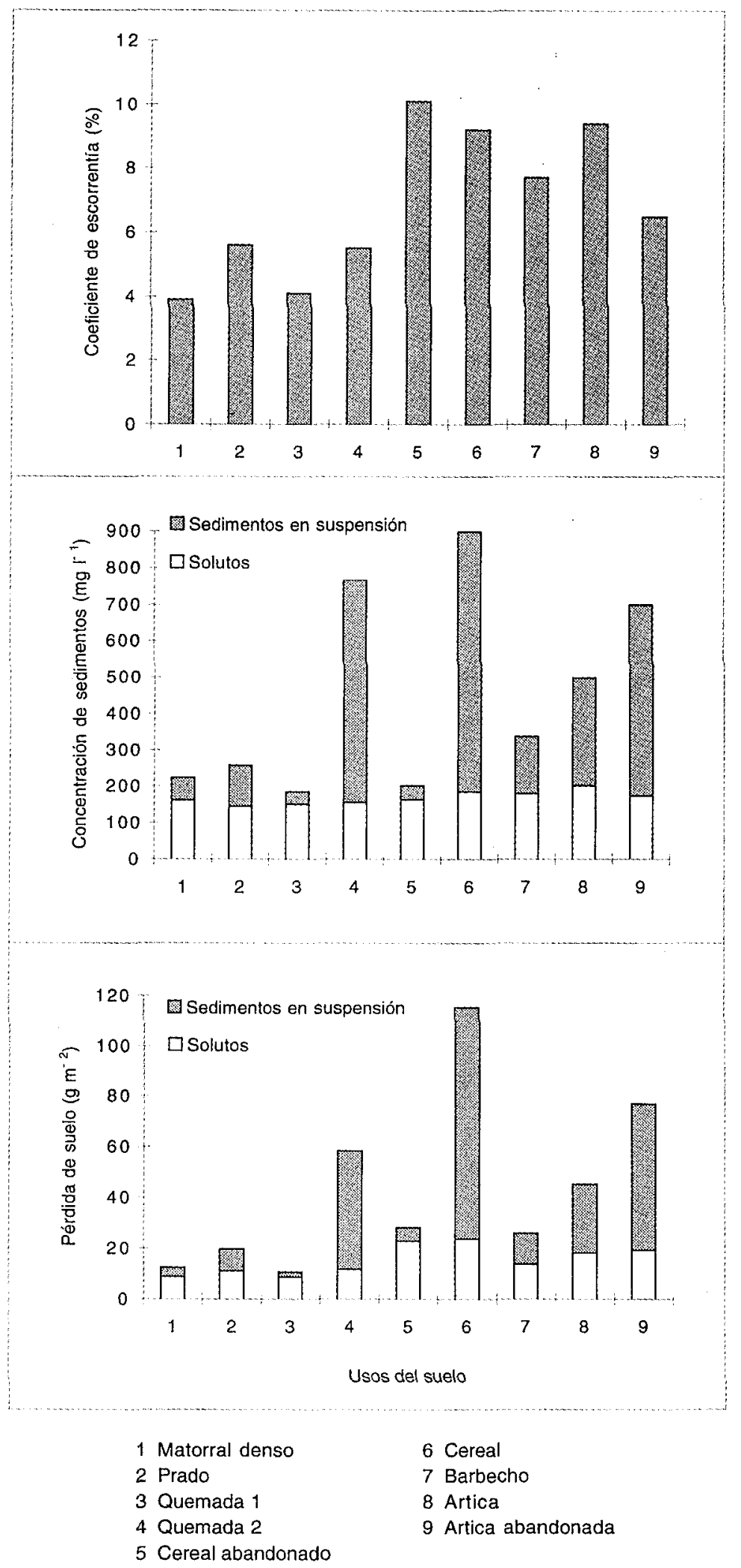

Fig. 4. Coeficientes de escorrentía, concentración de sedimento y pérdida de suelo en la Estación Experimental "Valle de Aísa" (1996). 
rápida recolonización vegetal debido a la fertilización con abono químico mientras estuvo en cultivo. En todo caso, los resultados confirman que la cubierta herbácea frena la erosión pero no tanto la escorrentía.

iii) La parcela de matorral quemado en 1993 registra bajos coeficientes de escorrentía (entre 5.5 y 8.6), pero concentraciones de sedimento relativamente altas (entre 432 y $767 \mathrm{mg.l}^{1}$ ), por lo que las pérdidas de suelo se sitúan en una posición intermedia (de 42.2 a $58.4 \mathrm{~g} \cdot \mathrm{m}^{-2}$ ) en comparación con el resto de los usos del suelo. La existencia de pequeños claros aún no colonizados por la vegetación explica las elevadas concentraciones.

iv) Las parcelas cultivadas (cereal con abono químico, artica y barbecho), así como la parcela de artica abandonada presentan coeficientes de escorrentía relativamente altos, al igual que las concentraciones y las pérdidas totales de suelo. Su comportamiento interanual es más irregular que en el resto de los usos, especialmente en el barbecho y el cereal con abono químico. Esto puede deberse al estado del suelo y la densidad de cubrimiento del cereal en el momento en que se registran las precipitaciones más intensas. Así, en el barbecho la rugosidad del suelo disminuye con el paso del tiempo (siendo baja en enero y alta en febrero, después del paso del arado), lo que condiciona mucho la infiltración y la concentración de sedimentos. En el caso del cereal el cubrimiento es muy elevado en los meses de mayo y junio, pero muy bajo en los meses posteriores a la siembra y después de la siega. Este efecto es menor en la parcela de artica, donde el cereal alcanza una densidad muy baja. Por su parte, la artica abandonada tiene un comportamiento muy parecido a la artica cultivada, ya que se encuentra en una fase muy inicial de la colonización vegetal, necesariamente lenta además como consecuencia del empobrecimiento del suelo después de cuatro años de cultivo.

En casi todas las parcelas noviembre y diciembre de 1996 representaron alrededor del 35 ó el 40\% de las salidas totales de sedimento en suspensión y de solutos, especialmente diciembre, debido al efecto de la saturación del suelo. En cambio, noviembre de 1997 representó entre el 10 y el 20\% del total.

Las tablas 1 y 2 incluyen información sobre la contribución relativa del sedimento en suspensión y de los solutos en las pérdidas totales en 1996 y 1997 respectivamente. Es interesante comprobar que las parcelas con bajos valores de erosión del suelo presentan mayores pérdidas en solutos que en sedimento en suspensión. Por otro lado, en las parcelas con elevadas pérdidas de suelo el sedimento en suspensión contribuye mucho más que los solutos a las cifras totales. Entre el material disuelto, el bicarbonato y el calcio representan más del $50 \%$ de las salidas totales de solutos. En las parcelas poco perturbadas (matorral denso, matorral incendiado en 1991 y prado) apenas registran pérdidas en los nutrientes más importantes $\left(\mathrm{Mg}^{2}+, \mathrm{K}+, \mathrm{Na}+\right.$ y $\left.\mathrm{NO}_{3}-\right)$, confirmándose 
LA GESTIÓN DE LOS USOS DEL SUELO COMO ESTRATEGIA PARA MEJORAR LA PRODUCCIÓN
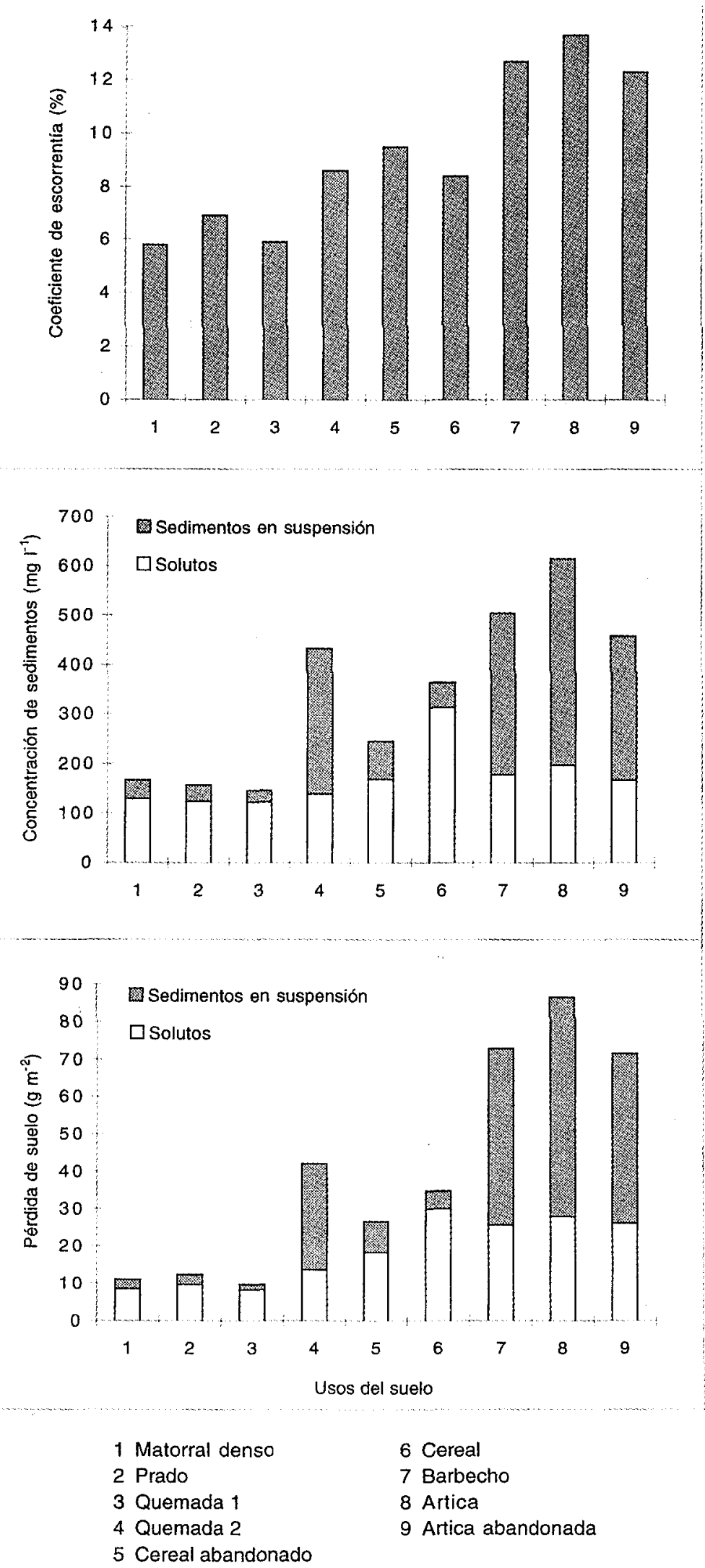

Fig. 5. Coeficientes de escorrentía, concentración de sedimento y pérdida de duelo en là Estación Experimental "Valle de Aísa" (1997). 
que una densa cubierta de matorral o de pasto controla muy bien la exportación de nutrientes. Por el contrario, en las parcelas labradas la proporción de bicarbonato y de calcio disminuye, mientras se incrementa la salida del resto de nutrientes.

Tabla 1. Escorrentía, solutos y sedimento en suspensión en diferentes usos del suelo (1996)

\begin{tabular}{lrrrrrr}
\hline & $\begin{array}{c}\text { Coef. } \\
\text { Escorr. } \\
\text { (\%) }\end{array}$ & $\begin{array}{c}\text { Concentración de } \\
\text { sedimento }\left(\mathrm{mg} . \mathrm{l}^{-1}\right)\end{array}$ & Pérdida suelo $\left(\mathrm{g} . \mathrm{m}^{-2}\right)$ & \\
& Solutos & $\begin{array}{c}\text { Sedim. } \\
\text { suspensión }\end{array}$ & Solutos & $\begin{array}{r}\text { Sedim. } \\
\text { suspensión }\end{array}$ & Total \\
\hline Matorral denso & 3.9 & 162 & 62 & 9.0 & 3.4 & 12.4 \\
Prado & 5.6 & 145 & 113 & 11.2 & 8.7 & 19.9 \\
Parcela quemada 1 & 4.1 & 152 & 34 & 8.8 & 2.0 & 10.8 \\
Parcela quemada 2 & 5.5 & 156 & 611 & 11.9 & 46.5 & 58.4 \\
Cereal abandonado & 10.1 & 164 & 38 & 23.0 & 5.3 & 28.3 \\
Cereal fertilizado & 9.2 & 186 & 712 & 23.9 & 91.4 & 115.3 \\
Barbecho & 7.7 & 180 & 158 & 13.9 & 12.2 & 26.1 \\
Artica & 9.4 & 202 & 297 & 18.3 & 26.9 & 45.2 \\
Artica abandonada & 6.5 & 175 & 524 & 19.3 & 57.6 & 76.9 \\
\hline
\end{tabular}

* Sólo durante diez meses (Marzo-Diciembre, 1996).

Finalmente, los resultados de la cuenca experimental Loma de Arnás muestran que los caudales reaccionan bruscamente frente a las precipitaciones. Durante tormentas cortas e intensas la carga de fondo tiene una gran importancia en el balance de sedimentos, llegando a representar más del $50 \%$ del transporte total (González et al., 1997). En cambio, a escala mensual o anual los solutos ( $250 \mathrm{mg} . \mathrm{l}^{-1}$ en promedio) y el sedimento en suspensión contribuyen en mayor medida a la exportación total de la cuenca ( $44 \%$ y $51 \%$ de la salida de sedimentos respectivamente en 1997, frente al 5\% de la carga de fondo) (Arnáez et al., 1998). Los primeros resultados confirman la importancia del cauce y de las áreas próximas en el balance de sedimentos de la cuenca Loma de Arnás (González et al., 1997). La cartografía de las fuentes de sedimentos demuestra que en las últimas décadas (al menos desde 1957) las áreas de contribución de sedimento han experimentado una contracción espacial, debido al abandono agrícola y a la recolonización vegetal (Molinillo et al., 1997). 
LA GESTIÓN DE LOS USOS DEL SUELO COMO ESTRATEGIA PARA MEJORAR LA PRODUCCIÓN

Tabla 2. Escorrentía, solutos y sedimento en suspensión en diferentes usos del suelo (1997)

\begin{tabular}{|c|c|c|c|c|c|c|}
\hline & \multirow{2}{*}{$\begin{array}{l}\text { Coef. } \\
\text { Escorr. } \\
(\%)\end{array}$} & \multicolumn{2}{|c|}{$\begin{array}{l}\text { Concentración de } \\
\left.\text { sedimento (mg. } .^{-1}\right)\end{array}$} & \multicolumn{2}{|c|}{ Pérdida suelo (g. $\left.\mathrm{m}^{-2}\right)$} & \multirow[b]{2}{*}{ Tota } \\
\hline & & Solutos & $\begin{array}{l}\text { Sedim. } \\
\text { suspensión }\end{array}$ & Solutos & $\begin{array}{l}\text { Sedim. } \\
\text { suspensión }\end{array}$ & \\
\hline Matorral denso & 5.8 & 130 & 37 & 8.6 & 2.5 & 11.1 \\
\hline Prado & 6.9 & 124 & 33 & 9.8 & 2.6 & 12.4 \\
\hline Parcela quemada 1 & 5.9 & 123 & 23 & 8.3 & 1.5 & 9.8 \\
\hline Parcela quemada 2 & 8.6 & 140 & 292 & 13.7 & 28.5 & 42.2 \\
\hline Cereal abandonado & 9.5 & 169 & 77 & 18.3 & 8.3 & 26.6 \\
\hline Cereal fertilizado & 8.4 & 314 & 50 & 30.0 & 4.7 & 34.7 \\
\hline Barbecho & 12.7 & 178 & 326 & 25.7 & 47.2 & 72.9 \\
\hline Artica & 13.7 & 198 & 416 & 27.9 & 58.6 & 86.5 \\
\hline Artica abandonada & 12.3 & 167 & 291 & 26.1 & 45.4 & 71.5 \\
\hline
\end{tabular}

\subsection{Futuros escenarios en la economía del agua frente a posibles cambios de uso del suelo}

A corto plazo no son previsibles nuevos cambios de uso del suelo en áreas de montaña submediterrénea, al menos de manera generalizada. La superficie cultivada continuará restringida a los fondos de valle y, debido a razones climáticas, sociales y económicas, es casi imposible imaginar otro cultivo distinto de los prados. En la actualidad el desequilibrio entre recursos de verano y de invierno (resuelto en el pasado con la trashumancia) obliga a dedicar toda la superficie cultivada a la producción de forraje para la alimentación del ganado durante 8 meses. En ese tiempo las laderas medias y bajas, ocupadas por campos abandonados, matorral y bosque, apenas son visitadas por el ganado, aportando muy poco a la dieta alimentaria. Dado que los censos de ganado se han adaptado, tras la crisis del sistema trashumante, a la capacidad de producción del espacio cultivado, los pastos de verano están claramente desaprovechados. De hecho, Remón \& Alvera (1989), al estudiar los pastos supraforestales del valle de Aísa, comprobaron que las laderas y fondos de valle más accesibles sólo son aprovechados en una $78 \%$, mientras el resto no es utilizado casi nunca.

La única posibilidad para reducir el desequilibrio estacional de recursos pastorales -y aumentar así el número de ovejas y vacas- se basa en recuperar algunos de los campos abandonados, ahora colonizados por matorral, y transformarlos en prados de siega o de diente. Los resultados obtenidos en la Estación Experimental "Valle de Aísa" demuestran que el comportamiento 
hidromorfológico de los prados es bastante parecido al de las parcelas con matorral denso, si bien registran pérdidas de suelo ligeramente superiores y coeficientes de escorrentía más elevados. En otros estudios previos, con parcelas experimentales de tamaño reducido, Ruiz Flaño et al. (1991) confirmaron los resultados presentados en este trabajo. De esta forma, una sustitución de matorrales por prados en algunos campos abandonados no debería incrementar necesariamente la pérdida actual de materiales disueltos y particulados.

En este caso el problema consiste en seleccionar los campos abandonados que podrían transformarse en prados. Lasanta (1988) y Ruiz Flaño (1993) argumentan que los campos en laderas cóncavas o en rellanos colgados con pendientes inferiores al 25\%, fueron los últimos en abandonarse, debido a que poseen una mayor productividad: es evidente que la topografia condiciona la organización espacial de la humedad del suelo, de los nutrientes y de los suelos más profundos. El cese del cultivo en muchos de estos campos no se debe a sus bajos rendimientos ni a que presenten problemas erosivos, sino a limitaciones de accesibilidad (ausencia de pistas y/o alejamiento de los pueblos). Mediante el empleo de un Modelo Digital del Terreno y un Sistema de Información Geográfica (Arc/Info V 7.04) ha sido posible identificar campos abandonados localizados en concavidades, por debajo de $1.600 \mathrm{~m}$ s.n.m. (es decir, el límite altitudinal para cualquier cultivo en el área de estudio) y con menos del $25 \%$ de pendiente (tabla 3). De las 1456 ha. abandonadas, las condiciones topográficas indican que 292 ha. son recuperables, es decir el 20\% del actual espacio abandonado. Proporciones similares podrían obtenerse en otros valles pirenaicos. Considerado la productividad de los prados dada por diferentes autores en este ambiente (Chocarro, 1990; Maestro et al, 1990), podrían alimentarse alrededor de 850 ovejas más durante 3 meses, lo que representa un aumento del $25 \%$ respecto al censo total ganadero existente en la actualidad en el valle de Aísa.

Tabla 3. Distribución de campos abandonados en el valle de Aísa, según forma de la ladera y porcentaje de pendiente.

Forma de la ladera

Cóncava

Llana

Convexa

Total abandonado

Total recuperable
Pendiente de la ladera Superficie (ha)

164,8

367,4

127,6

193,9

178,8

423,1

1455,6

292,4 


\section{Discusión y conclusiones.}

Experimentos llevados a cabo en el Pirineo Central español confirman que los diferentes usos del suelo dan lugar a una amplia gama de coeficientes de escorrentía y de producción de sedimento. Por ello es por lo que la gestión del territorio puede ser un sistema eficaz para mejorar la calidad y distribución estacional de los recursos hídricos, aumentando la infiltración y limitando la pérdida de sedimentos.

La mayoría de las áreas de montaña del mundo han experimentado importantes cambios de uso del suelo en las últimas décadas (Stone, 1992; Price, 1995). En las montañas europeas y particularmente en el Pirineo el sistema de gestión tradicional de las laderas bajas se basaba en el cultivo de cereales en laderas solanas, pastoreo intensivo en primavera y otoño e incendios periódicos del matorral. Desde un punto de vista geomorfológico los efectos de esta actividad fueron un incremento de la erosión y de la pedregosidad del suelo (Ruiz Flaño et al, 1992), el desarrollo o reactivación de varios conos de deyección (Gómez Villar, 1996) y la torrencialidad de muchos cauces fluviales (Martínez Castroviejo et al., 1991). A principios del siglo XX la mayoría de los ríos y barrancos mostraban una gran actividad, construyéndose muchas obras de restauración y protección (presas de retención de sedimentos) con el fin de reducir el transporte de carga de fondo.

A escala de cuenca, Loma de Arnás es un buen ejemplo de ambiente severamente perturbado, donde casi todas las laderas fueron cultivadas y donde sólo existen pequeños restos de la cubierta forestal, las avenidas son relativamente frecuentes y la carga de fondo puede contribuir ocasionalmente en una gran proporción a la entrega total de sedimentos de la cuenca. La erosión del suelo, y la torrencialidad fueron probablemente el problema ambiental más importante durante el llamado sistema tradicional de uso del suelo (García Ruiz et al., 1996).

A escala más detallada, las parcelas experimentales confirman que el cultivo de laderas pendientes tuvo consecuencias muy negativas para la conservación del suelo. La agricultura nómada produjo grandes cantidades de sedimentos debido a su baja productividad. El barbecho, en alternancia con el cereal, también causó mucha erosión debido a la baja densidad de la cubierta vegetal. Las parcelas cultivadas tuvieron también elevadas pérdidas de nutrientes causadas por la movilización de fertilizantes químicos u orgánicos añadidos previamente. Además algunas laderas de matorral eran frecuentemente quemadas, generando un súbito aumento de la erosión durante algunos meses, empobreciendo el contenido de nutrientes del suelo y, después de varios incendios repetidos, dificultando la colonización vegetal (Ruiz Flaño et al., 1992). 
El abandono agrícola ha tenido importantes consecuencias hidrológicas. La mayoría de los campos abandonados fueron rápidamente colonizados por comunidades herbáceas y, más tarde, por matorrales, reduciendo rápidamente la escorrentía superficial y la concentración de sedimento. Ruiz Flaño (1993) demostró que después de varios años de colonización por el matorral mejoraban algunas de las características edáficas de los campos abandonados (contenido en materia orgánica, porosidad, capacidad de cambio, contenido en nitrógeno). En el mismo sentido, la sustitución de cereales por prados en el área cultivada representa un claro descenso en la producción de sedimento y, probablemente con menor intensidad, en la escorrentía superficial. Un posible reemplazamiento del matorral denso por prados, en lugares adecuados tales como laderas cóncavas de pendiente suave, conducirá a un ligero incremento en la escorrentía superficial sin serios problemas de erosión a largo plazo.

La conclusión más importante de este trabajo es que los cambios de uso del suelo en el espacio agrícola durante el siglo XX representan una mejoría desde el punto de vista de la conservación del suelo. El abandono de tierras y la sustitución del cereal por prados contribuyen a controlar la erosión de las laderas y la torrencialidad en los canales fluviales. De hecho las fuentes de sedimentos ocupan cada vez menos superficie y las estructuras sedimentarias de los cauces tienden a la colonización y a la estabilización (Martínez Castroviejo et al., 1991). Aunque la influencia de fluctuaciones climáticas no debe ser ignorada, el descenso en la tasa de sedimentación en embalses pirenaicos desde 1965 podría relacionarse con esta evolución de los usos del suelo (Valero et al., 1997). De igual forma, los conos aluviales han reducido su actividad y los sectores funcionales ocupan áreas muy restringidas (Gómez Villar, 1996).

Una segunda conclusión es que la expansión generalizada del matorral supone una disminución del agua superficial disponible en la cuenca, debido tanto al efecto de la intercepción como de la infiltración. Las descargas estarán, pues, mejor reguladas, pero en conjunto los recursos de agua serán menores.

Esta es una cuestión muy importante, ya constatada por otros autores en el área mediterránea (Llorens, 1993; Llorens et al., 1997; Romero et al., 1988) y sólo puede resolverse mediante una adecuada gestión de los usos del suelo. En el ejemplo que se ha desarrollado en el presente trabajo ha podido comprobarse que la sustitución del matorral por prados en los mejores campos abandonados no sólo puede aumentar la cantidad de agua sino también incrementar el aprovechamiento ganadero, reducir los desequilibrios estacionales y contribuir a diversificar el paisaje. 


\section{Agradecimientos}

Este trabajo se ha elaborado con el apoyo de los proyectos "Producción de sedimentos y escorrentía como consecuencia de los cambios de uso del suelo en áreas de montaña" (AMB 96-040) y "EPROHIDRO: Estaciones permanentes para el estudio de los procesos hidrológicos en ambientes mediterráneos" (HID98-1056-C02-01), ambos financiados por la CICYT, y "Validating hydrological models using process studies and internal data from research basins" (VAHMPIRE, ENV4-CT95-0134) y "Water resources management in a changing environment: the impact of sediment on sustainability" (WARMICE, PL983156), financiados por la Comisión Europea

\section{Referencias bibliográficas}

Arnáez, J., García-Ruiz, J.M., Martí, C., Bordonaba, A., Errea, M.P. \& White, S. (1998): Avenidas y transporte de sedimentos en una pequeña cuenca de montaña media del Pirineo Central. In Investigaciones recientes de la Geomorfología española (A. Gómez-Ortiz \& F. Salvador, eds.), Universidad de Barcelona, pp. 161-170, Barcelona.

Bosch, J.M. \& HewletT, J.D. (1982): A review of catchment experiments to determinate the effect of vegetation changes on water yield and evapotranspiration. Journal of Hydrology, 55: 3-23.

Chocarro, C. (1990): Estudios ecológicos sobre los prados de siega del Pirineo central español: composición florística, producción y calidad. Tesis Doctoral. Universidad de Navarra: 344 pp. + anexos.

Dunne, T. \& LEOPOLD, L.B. (1978): Water in environmental planning. Freeman, 818 pp., San Francisco.

Francis, C.F. \& Thornes, J.B. (1990): Runoff hydrographs from three Mediterranean vegetation cover types. In Vegetation and erosion (J.B. Thornes, ed.), Wiley, pp. 363-384, Chichester.

García-Ruiz, J.M. \& LASANTA, T. (1993): Land-use conflicts as a result of land-use changes in the Central Spanish Pyrenees. A review. Mountain Research and Development, 13(3): 213-223.

García-Ruiz, J.M. \& VALERO-GARCÉs, B.L. (1998): Historical geomorphic processes and human activities in the Central Spanish Pyrenees. Mountain Researcb and Development, 18(4): 309-320.

García-Ruiz, J.M., Lasanta, T., González, C., Martí, C., White, S. \& Ortigosa, L. (1997): Sediment sources during the traditional land-use system in the 
Spanish Pyrenees. Physics and Chemistry of the Earth, 22 (3-4): 351-354.

García-Ruiz, J.M., Lasanta, T., Ortigosa, L., Ruiz-Flaño, P., Martí, C. \& GONZÁLEZ, C. (1995): Sediment yield under different land uses in the Spanish Pyrenees. Mountain Research and Development, 15(3): 229-240.

García-Ruiz, J.M., Lasanta, T., Ruiz-Flaño, P., Ortigosa, L., White, S., González,

C. \& MARTí, C. (1996): Land-use changes and sustainable development in mountain areas: a case study in the Spanish Pyrenees. Landscape Ecology, $11(5) ; 267-277$.

Gómez-VILLAR, A. (1996): Dinámica de conos aluviales en pequeñas cuencas torrenciales de montaña. Geoforma Ediciones, 191 pp., Logroño.

González, C., García-Ruiz, J.M., Martí, C., White, S., Errea, M.P. \& Arnáez, J. (1997): Sediment sources in a small, abandoned farmland catchment, Central Spanish Pyrenees. Pbysics and Cbemistry of the Earth, 22 (3-4): 291-293.

LASANTA, T. (1988): The process of desertion of cultivated areas in the Central Spanish Pyrenees. Pirineos, 132: 15-36.

LASANTA, T. (1989): Evolución reciente de la agricultura de montaña: el Pirineo aragonés. Geoforma Ediciones: 220 pp., Logroño.

LloRENS, P. (1993): Hydrological implications of afforestation of abandoned lands: water balance simulation of a small Mediterranean mountain basin. Acta Geologica Hispanica, 28: 131-138.

Llorens, P., LATron, J. y GALlarT, F. (1992): Analysis of the role of agricultural abandoned terraces on the hydrology and sediment dynamics in a small montainous basin (High Llobregat, Eastern Pyrenees). Pirineos, 139: 27-46.

Llorens, P., Poch, R., Latron, J. \& Gallart, F. (1997): Rainfall interception by a Pinus sylvestris forest patch overgrown in a Mediterranean mountainous abandoned area. I. Monitoring design ans results down to the event scale. Journal of Hydrology, 199: 331-345.

Maestro, M., Ferrer, C., Amella, A., Broca, A. \& Ascaso, J. (1990): Praderas naturales de secano de los fondos de valle del Pirineo Central (Huesca): Suelo, manejo, producción y calidad. Actas de la XXX Reunión Científica de la SEEP, pp. 176-183, San Sebastián.

Martínez-Castroviejo, R., Gómez-Villar, A. \& García-Ruiz, J.M. (1991): Ajustes fluviales derivados de cambios de usos del suelo en el Pirineo aragonés. Cuaternario y Geomorfología, 5; 91-106.

Molinillo, M., LASANTA, T. \& GarcíA-RuIz, J.M. (1997): Managing mountainous degraded landscapes after farmland abandonment in the Central Spanish Pyrenees. Environmental Management, 21(4): 587-598.

PRICE, M.J. (1995): Mountain research in Europe. UNESCO, The parthenon 
Publishing Group, 230 pp., Paris.

Remón, J.L. \& Alvera, B. (1989): Biomasa y producción herbácea en un puerto pirenaico de verano. Options Méditérranéennes, Série Séminaires, 3: 289292.

Romero-Díaz, A., López-Bermúdez, F., Thornes, J.B., Francis, C.F. \& Fisher, G.C. (1988): Variability of overland flow erosion rates in a semiarid Mediterranean environment under matorral cover, Murcia, Spain. Catena Suppl., 13: 1-12.

Rubio, V. \& HernándeZ, C. (1990): La evolución reciente del cauce del río Ara. Cuadernos de Investigación Geográfica, 16: 99-108.

Ruiz-Flaño, P. (1993): Procesos de erosión en campos abandonados del Pirineo. Geoforma Ediciones, 191 pp., Logroño.

Ruiz-Flaño, P., García-Ruiz, J.M. \& Ortigosa, L. (1992): Geomorphological evolution of abandoned fields. A case study in the Central Pyrenees. Catena, 1993-4): 301-308.

Rutz-Flaño, P., Lasanta, T., García-Ruiz, J.M. \& Ortigosa, L. (1991): The diversity of sediment yield from abandoned fields of the Central Spanish Pyrenees. IAHS Publ., 203: 103-110.

SLAYMAKER, O. (1991): The nature of geomorphic field experiments. In Field experiments and measurement programs in Geomorpbology (O. Slaymaker, ed.), Balkema, pp. 7-16, Rotterdam.

STONE, P.B. (1992): State of the world's mountains. Zed Books, 391 pp., London.

Valero-Garcés, B.L., Navas, A. \& Machín, J. (1997): Sediment deposition in the Barasona reservoir (Central Pyrenees, Spain): temporal and spatial variability of sediment yield and land use impacts. IAHS Publ., 245:241-249.

WALLING, D. (1991): Drainage basin studies. In Field experiments and measurement programs in Geomorpbology (O. Slaymaker, ed.), Balkema, pp. 17-60, Rotterdam. 
